\title{
A Rare Genetic Cause of End Stage Renal Disease- Nail Patella Syndrome
}

\author{
Azhar Ali Khan, Javaria Karamat
}

Department of Nephrology, Sheikh Zayed Hospital, Lahore, Pakistan

\begin{abstract}
:
Hereditary osteo-onchodysplasia syndrome commonly known as nail patella syndrome is an autosomal dominant disorder that manifests with skeletal abnormalities and renal disease. Its incidence is 1 in 50,000 and reported in patients all over world. The renal disease involves glomerular basement membrane (GBM) thickening and rarely proceeds to End stage renal disease (ESRD). It requires histological diagnosis with electron microscopy to differentiate from other disease associated with proteinuria. As no specific treatment is available so management involves early recognition and close follow up for symptomatic relief of renal as well as skeletal complications. Prevention involves genetic counselling being solely helpful. This case report is of a patient diagnosed with nail patella syndrome with strong family history but rapidly progressed to ESRD and we were not able to proceed for histology.
\end{abstract}

Keywords: Nail Patella Syndrome, Osteo-Onchodysplasia Syndrome, End Stage Renal Disease

\section{Corresponding Author}

Azhar Ali Khan

Department of Nephrology

Sheikh Zayed Hospital,

Lahore, Pakistan

Email: azhar_aq@hotmail.com

Received: June 16, 2020. Accepted Dec 3, 2020.

PJKD 2020;4(4):336-37

\section{Introduction:}

Nail Patella syndrome (NPS) involves mutation of LXB1 gene involved in limb and renal development. ${ }^{1}$ Renal disease occurs only in 30-40 percent and presents with sub nephrotic range proteinuria and microscopic haematuria with pathology being GBM thickening. ${ }^{2}$ No data available for long term renal prognosis but research conducted up till now reveals that it rarely progresses to $\operatorname{ESRD}(1-5 \%)$.

\section{Case Report:}

A 27 years old young gentleman presented in emergency with complains of shortness of breath and body swellings off and on 3 years back. He was newly diagnosed hypertensive with history of growth retardation. Previously he gives history of froth in urine for which he was never investigated. Family history reveals two brothers who died because of unexplained renal failure. His autoimmune history was negative, and he was never been on any nephrotoxic drug. His skeletal features were typical of nail patella syndrome with nail hypoplasia and digital abnormalities as shown in figure.

After clinical examination he was labelled as a case of nail patella syndrome and on further workup he had nephrotic range proteinuria with bilateral small sized kidneys and decreased eGFR, so we were not able to proceed for histological examination. He was evaluated by orthopaedic department and eye department as well which favoured our diagnosis. He was followed on regular basis with proteinuria and serum creatinine for next month but showed gradual deterioration of his GFR despite good blood pressure control. We had to initiate renal replacement therapy in the form of haemodialysis. Family history reveals two brothers who died because of his unexplained renal failure and one cousin who is currently under follow up with proteinuria and GFR measurements with same skeletal features.

\section{Discussion:}

NPS is caused by mutation in LMX1B gene which encodes a transcription factor that is found in podocytes and maintains integrity of actin skeleton. Diagnosis depends on clinical presentation and characteristic radiological findings with histology differentiating NPS nephropathy from others. Classical presentation is with skeletal abnormalities in the form of nail hypoplasia and digital abnormalities followed by patellar hypoplasia and pelvic abnormalities in order of incidence. Less common presentation is of renal disease, sensorineural hearing loss, glaucoma, vasomotor problems and other neurological manifestations. Nephropathy will present with mild proteinuria and microscopic haematuria mostly presenting at adolescence. Histological diagnosis can only be made on electron microscopy showing characteristic moth-eaten appearance in the form of multiple irregular lucent areas. Light microscopy and immunofluorescence are invariably normal. 


\section{Nail Patella Syndrome}

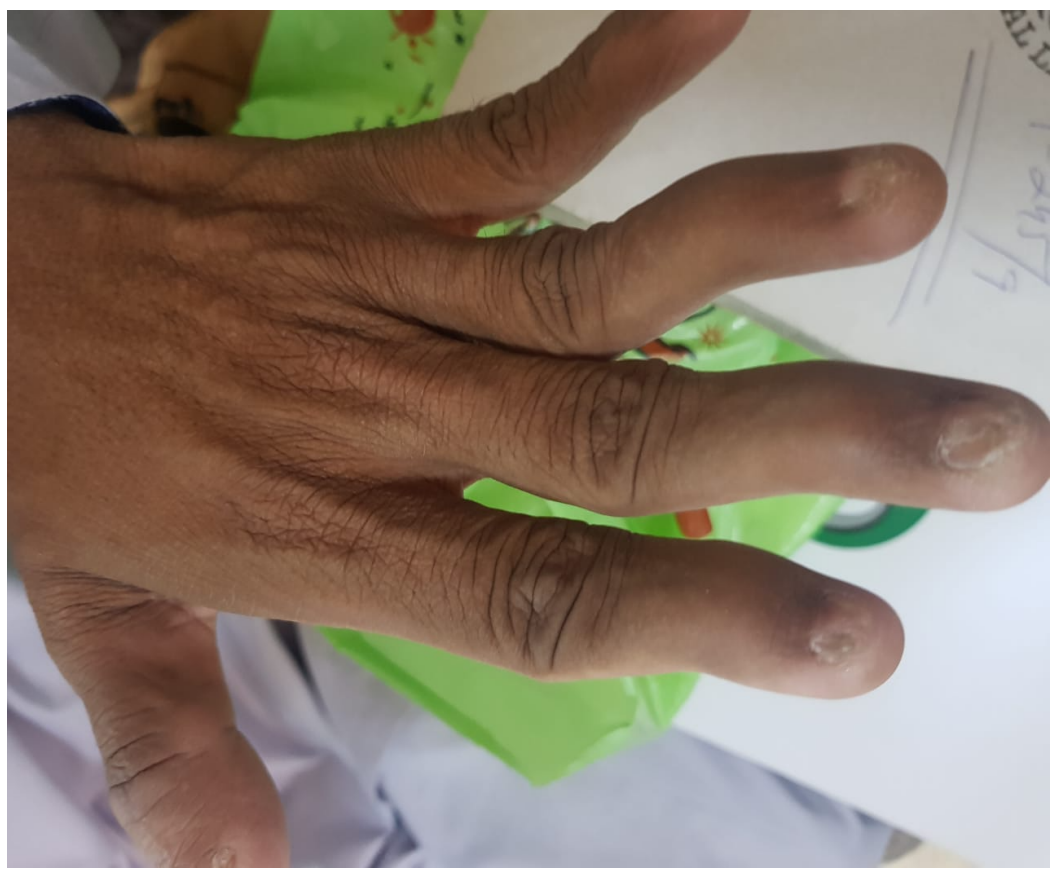

Figure: Nails showing hypoplasia and nail dystrophic changes, including discoloration, abnormal ridging and splitting, and triangular lunulae. Apparent is also swan neck deformity and loss of distal digital creases.

\section{Management:}

No specific therapy available however for nephropathy angiotensin converting enzyme inhibitors and angiotensin II receptor blockers are prescribed in those with proteinuria to halt progression. ${ }^{3}$ While orthopaedic management includes analgesia, physiotherapy, splinting, bracing or surgery.

\section{Transplantation:}

Renal replacement therapy in the form of transplant is an excellent option for 1 to $5 \%$ of patients who progressed to ESRD as no chances of recurrence in allograft observed.

\section{Prevention:}

Genetic counselling required for affected families as NPS has autosomal dominant inheritance. ${ }^{5}$

\section{Recommendations:}

Patients with proteinuria should be evaluated annually for hypertension and protein to creatinine ratio with early morning spot sample. NSAIDs for analgesia are best avoided with either ACE-I/ARBs are given to control protein excretion. If progressed to end stage than transplant is better option for renal replacement therapy. Genetic counselling being the only modality to decrease its incidence in affected families.

\section{Conclusion:}

Diagnosis of NPS is clinical however histology will help in distinguishing other GBM disorders. Nephropathy being benign rarely will progress to ESRD and renal transplant is best possible treatment option, however no specific therapy is there for treatment of skeletal and renal manifestations.

\section{Conflict of Interest: None declared}

\section{References:}

1. Bongers E. M., De Wijs I. J., Marcelis C., Hoefsloot L. H., \& Knoers N. V. Identification of entire LMX1B gene deletions in nail patella syndrome: evidence for haploinsufficiency as the main pathogenic mechanism underlying dominant inheritance in man. European Journal of Human Genetics 2008;16(10), 1240.

2. Granata A., Nori G., Ravazzolo R., Marini M., Castellino S., Sicurezza E., Fiore C.E., Mignani R. Nail-patella syndrome and renal involvement. Description of three cases and literature review. Clin Nephrol 2008;69(5), 377-382.

3. Proesmans W., Van Dyc, M., Devriendt K. Nail-patella syndrome, infantile nephrotic syndrome: complete remission with antiproteinuric treatment. Nephrol Dial Transplant 200924(4), 1335-1338.

4. Uranga V. M., Simmons R. L., Hoyer J. R., Kjellstrand C. M., Buselmeier T. J. Najarian, J. S. Renal transplantation for the nail patella syndrome. The American Journal of Surgery 1973;125(6), 777-779.

5. Looij B. J., Te Slaa R. L., Hogewind B. L, Van de Kamp, J. J. Genetic counselling in hereditary osteo-onychodysplasia (HOOD, nailpatella syndrome) with nephropathy. Journal Med Gen 1988;25(10), 682-686. 